\title{
Morphology and Surface Roughness of Cr-Coated Alternate Hard Disk Substrates for Ultra-High Density Magnetic Recording
}

\author{
Pawel GLIJER ${ }^{1}$, John M. SIVERTSEN 1 , Jack H. JUDY ${ }^{2}$ \\ The Center for Micromagnetics and Information Technologies (MINT) \\ 1) Department of Chemical Engineering and Materials Science, \\ 2) Department of Electrical Engineering, \\ University of Minnesota, Minneapolis MN 55455, USA
}

\begin{abstract}
Morphology and surface roughness of Canasite, SiC, carbon, glass disk and cover glass substrates covered with $0 \AA$, $500 \AA$ and $1000 \AA$ of $\mathrm{Cr}$ film were studied using an atomic force microscope (AFM). This study resulted in precise qualitative and quantitative descriptions of the sample surfaces. It was demonstrated that the surface morphology of a hard disk substrate determined the appearance and surface roughness of a thinner $\mathrm{Cr}$ film (500 $\AA$ ). Thicker $\mathrm{Cr}$ films (1000 $\AA$ ) controlled surface roughness, when deposited on smooth surfaces, with a regular surface morphology (carbon, glass disk, cover glass). Very deep (or very high) surface roughness features of $\mathrm{SiC}$ and Canasite surfaces, with diameters exceeding average $\mathrm{Cr}$ grain size, were not only reproduced but even enhanced by the $1000 \AA \mathrm{Cr}$ film.
\end{abstract}

\section{INTRODUCTION}

The rapid advancement of ultra-high density magnetic recording storage systems puts high demands on the design and quality of all the components. The hard disk substrate is one of the most important components. The physical properties of the substrate affect properties of a magnetic medium significantly. The surface roughness of the substrate influences friction, stiction and wear of the head-disk interface as well as magnetic anisotropy [1]. It is predicted that $10 \mathrm{Gbit} / \mathrm{inch}^{2}$ longitudinal recording may be realized in the near contact or contact regime [2]. Even higher densities may be achieved in contact perpendicular recording. Therefore the surface roughness of the magnetic medium will affect the effective physical and magnetic head-to-medium separation.

Several new materials for hard disk alternate substrates including carbon, glass, Canasite [3][4][5] and other ceramics have recently been used. The surface roughness of these materials is rarely discussed, however, and the authors usually limit their results to only one roughness parameter ( $\mathrm{Ra}$ or $\mathrm{Rms}$ ). In addition no extensive studies have been reported on correlating surface morphologies of hard disk substrates with the final surface roughness and morphology of a thin film media.

The purpose of this paper is to report on studies of the morphology and surface roughness of several alternate hard disk substrates covered with $\mathrm{Cr}$ thin films using atomic force microscopy (AFM).

\section{EXPERIMENTAL PROCEDURE}

Thin $\mathrm{Cr}$ films of $500 \AA$ and $1000 \AA$ were deposited on different substrates using a RF diode sputtering system with RF substrate bias at a deposition rate of $\sim 250 \AA / \mathrm{min}$, at $10 \mathrm{mtorr}$ Ar pressure and $-100 \mathrm{~V}$ substrate bias. The target-to-substrate distance was $2.5^{\prime \prime}$ and the average substrate temperature developed during deposition was $120^{\circ} \mathrm{C}-150^{\circ} \mathrm{C}$ as measured with a surface thermometer. These sputtering parameters were chosen to create high mobility deposition conditions so there was no tendency to extensively roughen the covered surface [6]. Small coupons cut from $2.5^{\prime \prime}(65 \mathrm{~mm})$ in diameter hard disk substrates of carbon, $\mathrm{SiC}$, glass and Canasite and cover glass were used as substrates for deposition. The crystallographic orientation of $\mathrm{Cr}$ films was determined using an $\mathrm{X}$-ray diffractometer.

Very high smoothness and good surface repeatability are the features characteristic of thin cover glass. Therefore $\mathrm{Cr}$ films deposited on these substrates were treated as reference samples, showing the kind of roughness and surface morphology developed by $\mathrm{Cr}$ films when substrate surface morphology effects are negligible.

The prepared samples as well as coupons with no $\mathrm{Cr}$ overcoat were studied in an atomic force microscope (AFM). Sample areas with diameter of $0.5 \mu \mathrm{m}$ to $7 \mu \mathrm{m}$ were imaged. The following surface roughness parameters were measured:

The mean roughness $R_{a}$ is defined [7] by

$$
R_{a}=\frac{1}{L_{x} L_{y}} \int_{0}^{L_{y}} \int_{0}^{L_{x}}|f(x, y)| d x d y
$$

where $f(x, y)$ describes surface position relative to the mean plane, $\mathrm{L}_{\mathrm{x}}, \mathrm{L}_{\mathrm{y}}$ are the dimensions of the surface; $\mathrm{Rz}$ - average difference in height between the five highest peaks and five lowest valleys relative to the mean plane.

The $\mathbf{R}_{\mathbf{a}}$ parameter describes surface in general by classifying it as "smooth" or "rough". $R_{z}$ is a measure of an average roughness amplitude and compared with $R_{a}$ indicates nonuniformities in the surface morphology. For this reason $\mathbf{R}_{\mathbf{Z}}$ is an important tribological parameter [8]. 
These roughness parameters were measured from the areas between $4 \mu \mathrm{m}^{2}$ and $12 \mu \mathrm{m}^{2}$ depending on what minimum area was necessary to include all high and low frequency surface features.

\section{RESULTS AND DISCUSSION}

Crystallographic texture of the $\mathrm{Cr}$ films developed with thickness in a similar manner for all the substrates used. The thinner films of $500 \AA$ had mixed orientation with $\{110\}$ dominant texture. The thicker films of $1000 \AA$ had strong $\{110\}$ crystallographic texture with only a few $\{100\}$ and $\{211\}$ oriented grains in the film.

Figure 1 shows surface roughness parameters for all of the samples examined and will be discussed in conjunction with AFM images shown in Figure 2 and 3. It is clear that the substrates used represent a wide range of surface roughness and morphologies:

a) Cover glass surface is very smooth $\left(R_{a}=3 \AA\right)$. Very flat terraces with diameter of about $4000 \AA$ and height of 6 to $10 \AA$ are characteristic for its surface morphology.

b) Carbon surface is almost as smooth as the cover glass $\left(\mathrm{R}_{\mathrm{a}}=5 \AA\right.$ ) but has a different morphology consisting of the very shallow grooves from fine polishing and a fine granularity. Granules, observed with diameters of $700 \AA$ $1500 \AA$ are the tops of several grains of nanocrystalline carbon merged together.

c) Glass disk surface $\left(R_{a}=10 \AA\right)$ is covered with shallow (up to $15 \AA$ ), wide grooves ( width of $800-1300 \AA$ ) and long ridges produced by polishing.

d) $\mathrm{SiC}$ surface morphology $\left(\mathrm{R}_{\mathrm{a}}=12 \AA\right)$ also consists of polishing grooves. Very deep (down to $40 \AA$ ) grooves as well as much more narrow, shallow ones could be distinguished on the surface.

e) Canasite $\left(R_{\mathrm{a}}=24 \AA\right.$ ) has an isotropic surface texture arising from polishing due to the differential hardness of crystalline and amorphous phases [5]. The surface consists of a large hills ( up to $13000 \AA$ wide and up to $150 \AA$ high) and wide, shallow valleys. Some hills have large craters on top with widths of $2000 \AA$ to $3000 \AA$ and depths up to $120 \AA$ giving them vulcano-like appearance.

Both roughness data (Fig. 1) and AFM micrographs (Fig. 2 and 3 ) show that surface roughness of $500 \AA$ thick $\mathrm{Cr}$ films was controlled by the roughness of the substrate. Both $\mathbf{R}_{\mathbf{z}}$ and $\mathbf{R}_{\mathbf{a}}$ increased for almost all the samples except Canasite $\left(R_{a}\right)$ and glass disk $\left(R_{z}\right)$. In case of Canasite small decrease in mean roughness was most probably caused by the nanoroughness of $\mathrm{Cr}$ film which made the surface more regular. Slight decrease in $R_{Z}$ for glass disk sample was most probably due to filling of shallower grooves by $\mathrm{Cr}$ grains. The surface morphology of the films deposited on rougher substrates (glass disk, $\mathrm{SiC}$, Canasite) was almost identical to that of uncoated substrates. Fine surface grains of $\mathrm{Cr}$ (200 to $400 \AA$ in diameter) were barely distinguishable in these samples. In case of the two smoothest samples surface grains of $\mathrm{Cr}$, $300 \AA$ to $500 \AA$ in diameter, were clearly visible. Grains grown on carbon are round, regular in shape; those grown on glass tend to be elongated which probably resulted from differences in initial surface state affecting grain nucleation.
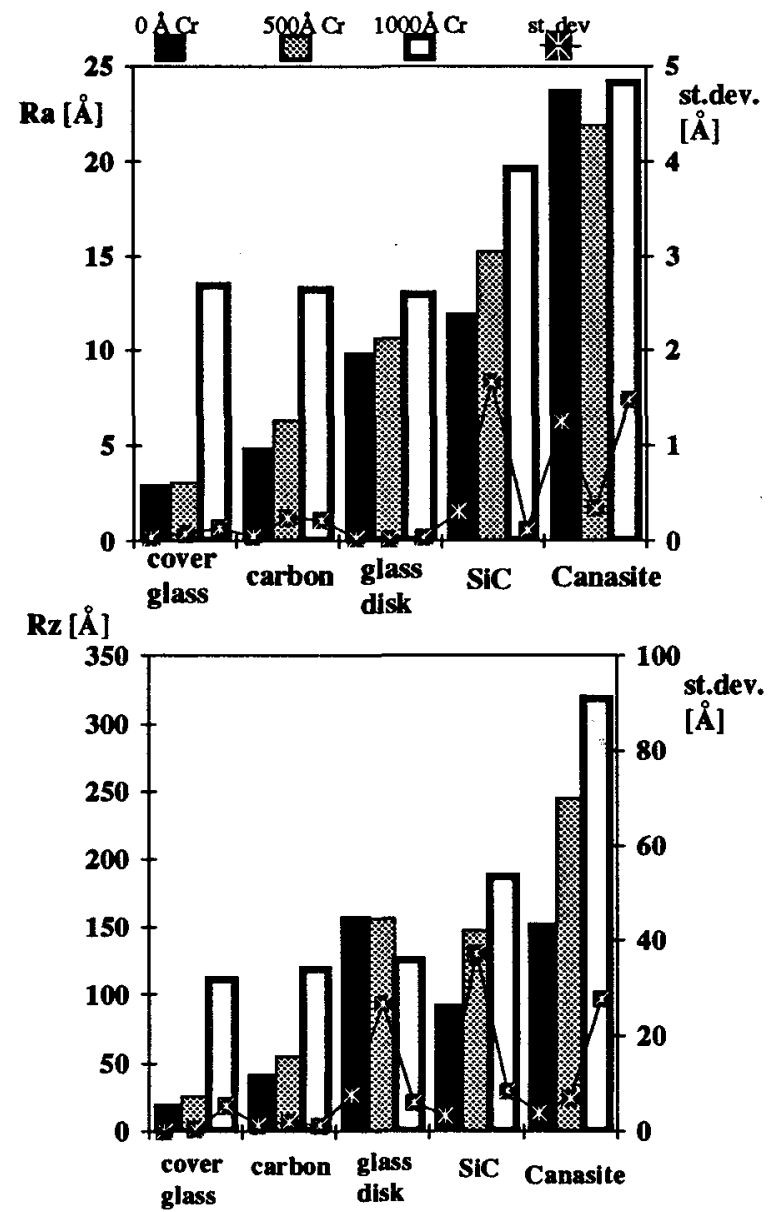

Fig. 1 Roughness ( $\mathrm{Ra}$ and $\mathrm{Rz}$ ) for examined samples. Each data point is an average of the three measurements obtained from different fields of view. Standard deviation of these three measurements (st.dev.) is also shown.

Increase of the $\mathrm{Cr}$ film thickness to $1000 \AA$ drastically changed the surface morphology and roughness of all the samples. From Fig. 1 it is obvious that surface roughness of $\mathrm{Cr}$ deposited on cover glass, carbon and glass disk is in these cases entirely controlled by the $\mathrm{Cr}$ film. Roughness parameters of all of these three samples are almost identical $\left(R_{a} \approx 13 \AA, R_{z}=112 \AA\right.$ to $\left.120 \AA\right)$. Elongated grains of $\mathrm{Cr}(300 \AA \times 700 \AA$ on average) are clearly visible on these surfaces. Slight differences in the average Cr grain sizes and shapes (see Fig. 2 and 3) are probably a result of different nucleation at disk surfaces and possible differences of substrate temperatures developed during deposition [6]. It should be noted that roughness 


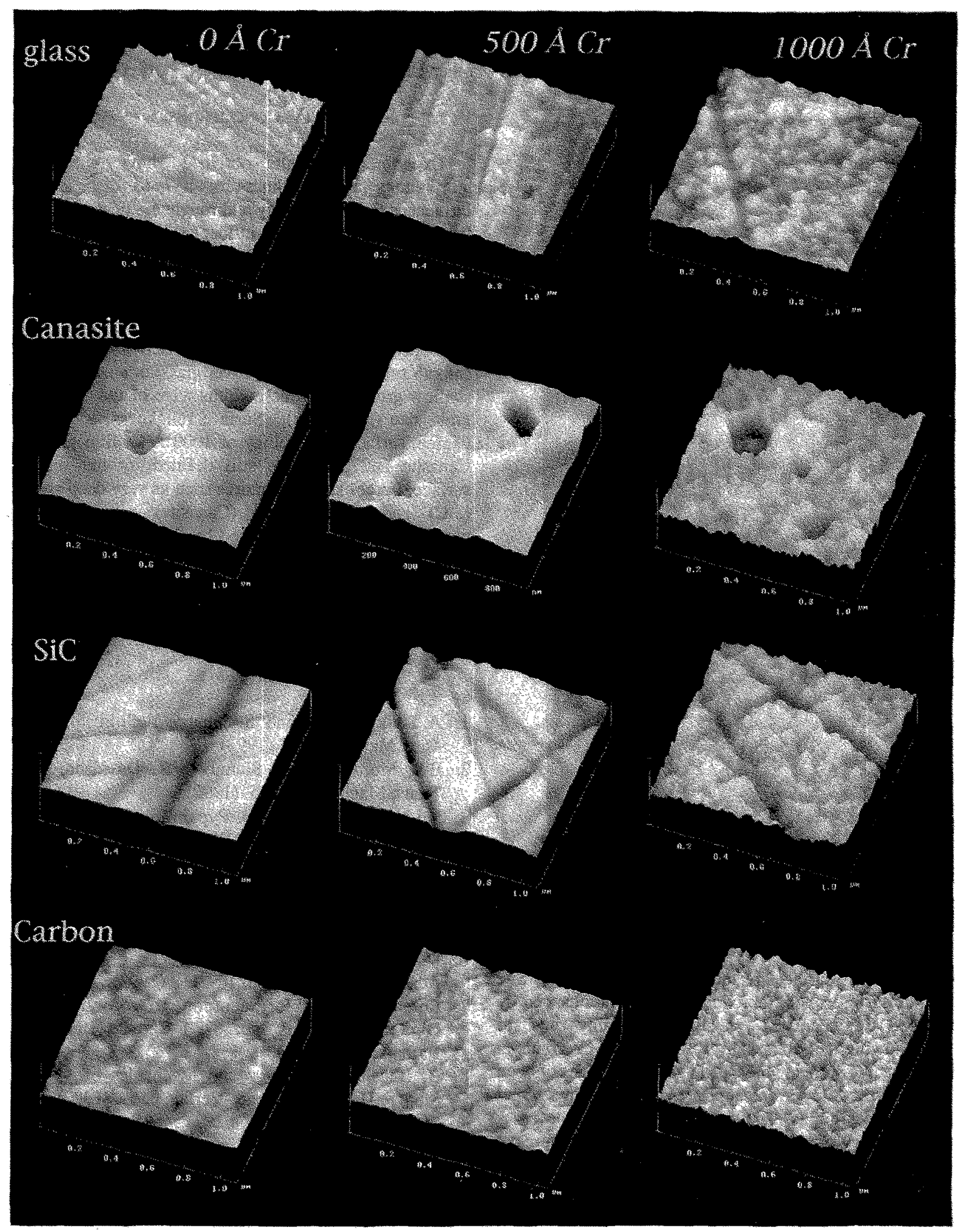

Fig. 2. AFM surface plots of $\mathrm{Cr}$ films deposited on altemate disk substrates. Views are $\sim 1.2 \mu \mathrm{m}$ in diameter. 
amplitude $R_{\mathrm{z}}$ decreased with the thickness of $\mathrm{Cr}$ when deposited on glass disk which was due to smoothing out of the shallow grooves and ridges on the glass surface. Some deeper grooves at the glass disk sample surface are still visible (see Fig. 2) and they cause slightly larger $\mathbf{R}_{\mathbf{z}}$ than for carbon and cover glass.

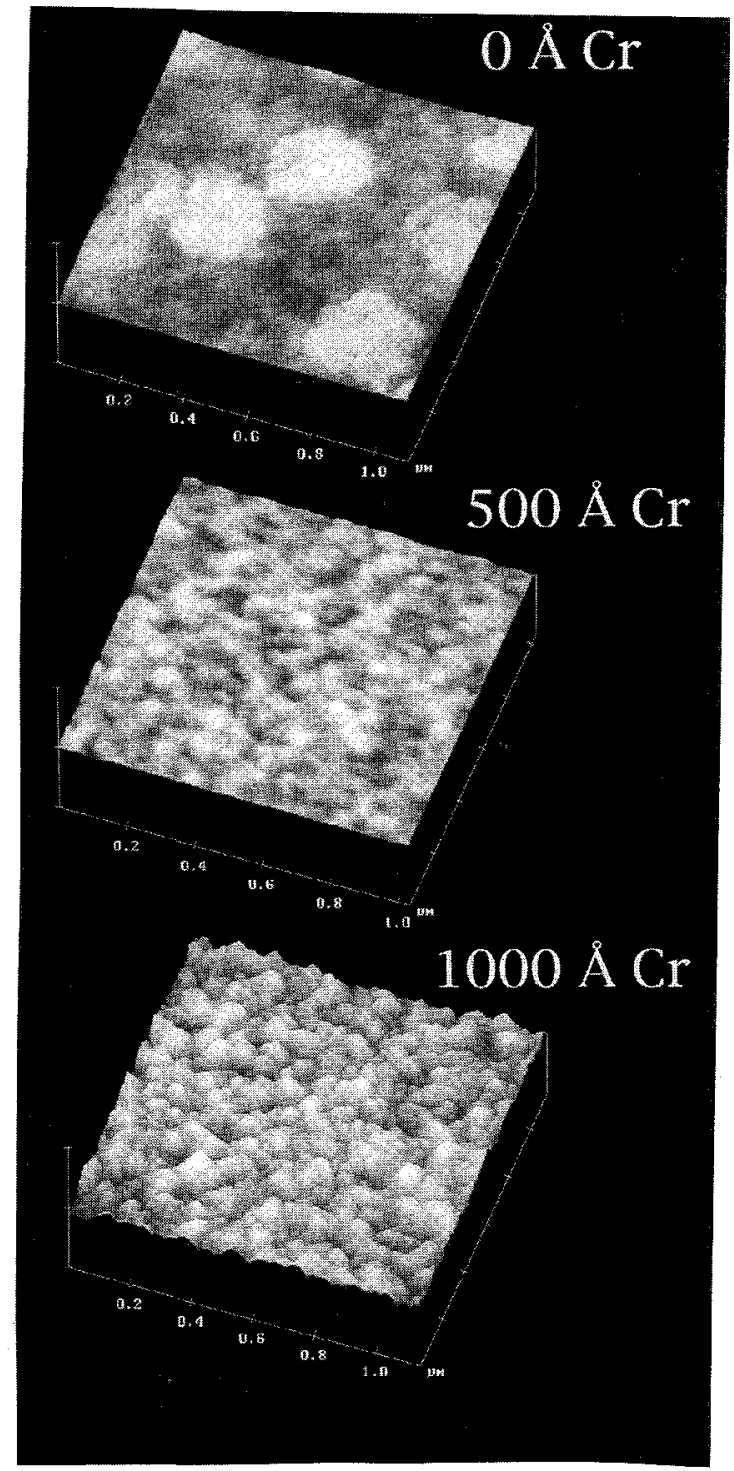

Fig. 3. AFM surface plots of $\mathrm{Cr}$ film deposited on cover glass

The larger surface features of Canasite and SiC substrate surfaces were reproduced and even enhanced by $1000 \AA \mathrm{Cr}$ deposited on them probably due to autoshadowing and abnormal growth rate of $\mathrm{Cr}$ grains at these spots. Cr surface grains growing at the edges of deep grooves at the SiC surface appear to be higher and larger than the other grains. The groove depths in some cases exceeded $80 \AA$. Shallower surface morphology features at the Canasite surface were covered by the $1000 \AA \mathrm{Cr}$ film which led to almost no change in the mean surface roughness compared to the uncoated sample. Larger features such as high hills and craters increased their height. Crater depths up to $400 \AA$ were measured in this sample. $\mathrm{Cr}$ grains at the crater edges have different appearance than at the other areas of the sample, where $\mathrm{Cr}$ grains resemble grains observed on the surfaces of the other samples.

\section{CONCLUSIONS.}

Experimental results obtained show that surface morphology and roughness of a hard disk substrate influences the morphology and roughness of the deposited film. The strength of this influence is determined mostly by the initial surface roughness features of a substrate and the film thickness. For thin films of $\mathrm{Cr}(500 \AA)$ surface morphology and roughness were to high degree controlled by a substrate. Surface roughness and morphology was controlled by the $1000 \AA \mathrm{Cr}$ film itself when grown on smooth substrates (carbon, cover glass) or a substrate with shallow grooves of which widths were of the order of $\mathrm{Cr}$ grain size (glass disk). Surface roughness, and to high degree, surface morphology of $1000 \AA \mathrm{Cr}$ film grown on Canasite and $\mathrm{SiC}$ were still defined by the substrate surface. Larger surface features such as very deep grooves (SiC) and deep craters (Canasite) were not only reproduced but even enhanced by the $\mathrm{Cr}$ film leading to large increase in surface roughness amplitude. The results also show that such extreme substrate surface features may lead to large microstructural nonuniformities in the overcoating film.

\section{REFERENCES}

1. A. Ishikawa, T. Ohno, S. Sakano, Y. Shiroshi, J. Mag. Mag. Mat., 120, 357 (1993)

2. E.S. Murdock, R.F. Simmons, R. Davidson, IEEE. Trans. Magn., 28, 3075 (1992).

3. D. Latev, V. Dorfman, B. Pypkin, Surface Coatings \& Technology, 47, 308 (1991).

4. S. Onodera, K. Ouchi, Y. Nakamura, S. Iwasaki, IEEE Transl. J. Mag. Jap., 7, 10 (1992).

5. H.-C Tsai, A. Eltoukhy, IEEE. Trans. Magn., 27, 5142 (1991).

6. M. Ohring, The Materials Science of Thin Films, Academic Press Inc., pp. 109-118 (1992).

7. Reference manual for Nanoscope III, software ver. 3.0, Digital Instruments Inc, 6780 Cartona Drv., Santa Barbara, CA 93117.

8. B. Bhushan, Tribology and Mechanics of Magnetic Storage Devices, Springer-Verlag, p. 69, (1990). 